Curr Opin Pulm Med. 2012 September ; 18(5): 441-446. doi:10.1097/MCP.0b013e328356d03c.

\title{
Biomarkers in idiopathic pulmonary fibrosis
}

\author{
Yingze Zhang and Naftali Kaminski \\ Division of Pulmonary, Allergy and Critical Care Medicine, Dorothy P. and Richard P. Simmons \\ Center for Interstitial Lung Disease, University of Pittsburgh, Pittsburgh, Pennsylvania, USA
}

\begin{abstract}
Purpose of review-This review examines the recent literature on molecular biomarkers of idiopathic pulmonary fibrosis (IPF). Specific attention is dedicated to the recent studies that identified the genes associated with IPF and the peripheral blood biomarkers that predict outcome in IPF.
\end{abstract}

Recent findings-Multiple studies attempted to identify diagnostic and predictive biomarkers in IPF. Until recently, these studies were limited in size and lacked replication, but still when taken together provided convincing evidence that changes in blood proteins (KL-6, SP-A, MMP-7, CCL-18, among others) or cells (fibrocytes and T-cell subpopulations) are indicative of the disease presence and outcome. More recently, larger studies have identified gene polymorphisms associated with IPF, as well as protein markers and integrated clinical and molecular prediction rules that accurately predict outcome in patients with IPF.

Summary-The peripheral blood contains disease presence and outcome relevant information, and suggests distinct biologically defined outcome trajectories in patients with IPF. Although recently identified biomarkers should still be validated in multiple clinical contexts, there is sufficient evidence to suggest that collection of peripheral blood biomarkers needs to be incorporated in the design of drug studies and that some of these markers be clinically evaluated in lung transplant prioritization.

\section{Keywords}

genomics; MMP-7; MUC5b; PCMI; personalized medicine

\section{INTRODUCTION}

Molecular biomarkers have rapidly become a buzz-word in pulmonary medicine. Clinical scientists and drug companies repeatedly mention that they are the most critical missing link in improving drug development in chronic lung disease in general, and idiopathic pulmonary fibrosis (IPF) in particular. The reason for this is quite obvious - IPF, a chronic, progressive

\footnotetext{
(c) 2012 Wolters Kluwer Health Lippincott Williams \& Wilkins.

Correspondence to: Naftali Kaminski, MD, University of Pittsburgh Medical Center, NW 628 MUH, 3459 5th Avenue, Pittsburgh, PA 15261, USA. Tel: +1 412624 7166; fax: +1 412624 1670; kaminskin@ upmc.edu.

Conflicts of interest

N.K. is an inventor on a patent application on the use of peripheral blood proteins to diagnose and predict outcome in IPF, as well as on the use of microRNAs in the management of IPF. N.K. is a consultant to Stromedix and Sanofti-Aventis, has industry sponsored grants from Gilead and Celgene, as well as multiple industry collaborations.
} 
and frequently lethal interstitial lung disease (ILD) of unknown origin [1,2], still presents a challenge in clinical research because of its variable and unpredictable course and the lack of easily reproducible surrogate endpoints for patient relevant outcomes [3]. In fact, it is highly common that speakers at international conferences and authors of reviews and position statements mention the dire need for biomarkers and bemoan the lack of reproducible and validated biomarkers [4",5"], often ignoring the emerging body of work suggesting that the peripheral blood is indicative of disease presence, stage and prognosis in IPF [6].

Molecular biomarkers can be defined as molecules or genes that carry information about the health or disease state of the individual assayed. Generally speaking, biomarkers can be divided into several classes based on the type of the information that they provide.

Diagnostic biomarkers allow the distinction of one disease from the other, and can be used in disease classification and diagnosis. Disease susceptibility markers - most often gene mutations and polymorphisms associated with the disease - are often included with diagnostic markers, but in fact differ because in the healthy individual they just indicate an increased risk and their diagnostic value is unclear in complex disease. Prognostic biomarkers are markers that allow the prediction of outcome, usually at the time of presentation. Diagnostic and prognostic markers should be distinguished from disease activity biomarkers that may change during the course of the disease - although in some cases they may overlap. The last group of biomarkers can be broadly defined as treatment efficacy biomarkers - these include markers that a drug is indeed affecting the pathway it is supposed to affect, markers that indicate toxicity and markers that indicate a real beneficial drug effect that could eventually be used as surrogate endpoints in drug studies.

In the current review, we focus mainly on peripheral blood biomarkers and only mention tissue and bronchoalveolar lavage fluid data when relevant. We present the recent data that supports the availability and feasibility of obtaining all previously mentioned classes of biomarkers, their relative importance as well as the steps we believe are required to move biomarkers in IPF into the clinical domain.

\section{DIAGNOSTIC BIOMARKERS}

It is the opinion of the authors of this review that diagnostic biomarkers are, at this stage, the least pressing question in biomarker discovery in IPF. The recent guidelines for diagnosis of IPF outline a clear path for diagnosis and it is yet unclear how complex is the differential diagnosis. However, it is important to mention that ruling out other conditions, potentially more responsive to immunosuppressive therapeutics, is critically important. In the context of peripheral blood markers, multiple molecules have been shown to distinguish patients with IPF from controls. These include KL-6, surfactant proteins SP-A and SP-D, matrix metalloproteases MMP-1 and MMP-7, SPP1 and YKL-40 (reviewed in [6]). The diagnostic utility of any of these molecules is in doubt as the majority of the studies usually only compared IPF to control individuals, and when smoking controls or other ILDs were analyzed, they quite often had increased levels of the markers [7-9]. The studies usually did not include replication cohorts. Rosas et al. [10] applied a targeted proteomic approach and identified a protein signature including MMP-1, MMP-7, MMP-8, IGFBP-1 and 
TNFRSA1F that distinguished patients with IPF from control individuals with a sensitivity of $98.6 \%$ and a specificity of $98.1 \%$. To determine the specificity of this signature, they demonstrated that concentrations of MMP-1 and MMP-7 were significantly higher in the plasma of patients with IPF compared with patients with chronic obstructive pulmonary disease (COPD) or sarcoidosis and differentiated IPF from hypersensitivity pneumonitis patients with a sensitivity of $96.3 \%$ and specificity of $87.2 \%$. They did not look at the other idiopathic interstitial pneumonias (IIPs) and it seems that at least MMP-7 is also increased in patients with non-specific interstitial pneumonia [11]. The authors replicated the results in an independent cohort and demonstrated that MMP-7 was increased in individuals with subclinical ILD compared to control individuals $(P=0.019)$ and significantly lower in fullblown IPF patients $(P<0.0001)$, suggesting that it could be used as potential marker for early detection. Taken together, it is obvious that while peripheral blood protein repertoire in IPF is clearly different from control individuals, the role of confounding factors such as smoking status, age and effects of chronic lung disease should be evaluated more systematically. One strategy is to directly profile tissues from patients with IPF, COPD and IIPs, and identify novel markers that are disease specific. Public availability of datasets such as the Lung Genomics Resource Consortium (LGRC) [12] that include parallel analysis of multiple chronic lung diseases as well as smoking and nonsmoking controls should allow the investigators to potentially identify novel markers that are disease specific.

\section{DISEASE SUSCEPTIBILITY MARKERS}

Multiple mutations associated with familial and sporadic forms of IPF have been reported including mutations in surfactant [13-15] and telomerase proteins [16,17]. Mushiroda et al. [18] also identified polymorphisms within TERT [single nucleotide polymorphism (SNP) in intron 2 of the TERT gene - rs2736100] in a genome-wide association (GWA) study including a derivation cohort of 159 sporadic IPF patients and 934 controls as well as a replication cohort of 83 sporadic IPF cases and 535 controls. Interestingly, leukocyte telomere shortening was found in $24 \%$ of familial pulmonary fibrosis and $23 \%$ of sporadic IPF cases when compared to control individuals $\left(P=2.6 \times 10^{-8}\right)$ [19] in a study that contained 201 control individuals, 59 probands with familial pulmonary fibrosis and 73 sporadic pulmonary fibrosis cases without TERT or TERC mutations. Similarly, Alder et al. [20] demonstrated that IIP patients had shorter telomeres when compared to age-matched controls in leukocytes and alveolar epithelial cells $(P<0.0001)$, suggesting the potential role of telomere shortening as a marker of increased risk of adult-onset pulmonary fibrosis. Other genetic variants have been described in IPF, including genes encoding ELMOD2 [21], IL-1 [22], CR-1 [23], IL12p40 and IFN-gamma [24], NOD2/CARD15 [25], MMP-1 [26], ENA-78, IP-10 and VEGF [27], CDI6b [28], IL-8 [29] and HER2 [30], but the majority have not been replicated. More recently, Seibold et al [31"'] identified a SNP in the putative promoter of $M U C 5 B$ (rs35705950) that was associated with familial interstitial pneumonia (minor allele frequency of $34 \%, P=1.2 \times 10^{-15}$ ) and IPF (minor allele frequency of $38 \%, P$ $\left.=2.5 \times 10^{-37}\right)$; in controls, the minor allele frequency was $9 \%$. The odds ratio was 6.2 [95\% confidence interval (CI) 3.7-10.4] for familial interstitial pneumonia and 8.3 (95\% CI 5.811.9) for IPF. The findings by Seibold and colleagues were simultaneously confirmed by us [32] in an independent case-control study that included 341 IPF and 801 control individuals. 
The minor-allele frequency was $34.3 \%$ in patients with IPF and $11.1 \%$ in controls (allelic association, $P=7.6 \times 10^{-40}$ ). The discovery of both rare mutations and common polymorphisms associated with IPF greatly improves the ability to screen the family members of patients with IPF, although additional studies are required to determine whether patients with distinct genetic predispositions exhibit distinct clinical manifestations.

\section{PROGNOSTIC BIOMARKERS}

This class of biomarkers is probably the most developed for IPF - in fact, it seems now evident that patients with IPF exhibit a biomarker profile that is indicative of outcome and contributes beyond the information that can be gleaned from the clinical presentation.

Selman et al. [33] introduced in 2007 the concept of different rates of progression in IPF that demonstrated different gene-expression patterns in the lungs, an observation later replicated by Boon and colleagues [34].

Recently, evidence has emerged in support of the peripheral blood as an informative source for outcome prediction at presentation. High blood concentrations of KL-6, also known as MUC-1, have been repeatedly shown to be predictive of decreased survival in IPF. Most of the studies are limited by cohort size and lack replication, but are still highly consistent and support the use of KL-6 in disease stratification [8]. Other notable studies include the work by Prasse et al. [35], who demonstrated in a prospective cohort of 72 patients that serum CCL18 levels were able to predict the outcomes in IPF, the demonstration by Kinder et al. [36] in a cohort of 81 patients that serum SP-A was a predictor of early mortality in IPF, and the recent demonstration by Korthagen et al. [37] in 79 patients that high serum concentrations of YKL-40 distinguished two groups with distinct survival patterns with the hazard ratio for serum YKL-40 (cut-off 79ng/ml) as 10.9 (95\% CI 1.9-63.8, $P<0.01$ ). Most recently, we applied a targeted proteomic approach and screened 95 proteins in the plasma of 140 IPF patients (derivation cohort) and validated the results in a replication cohort (101 patients) [38"']. High plasma concentrations of MMP-7, ICAM-1 and IL-8 were predictive of poor overall survival in both cohorts. We used the derivation cohort to derive a personal clinical and molecular mortality prediction index (PCMI) using the step AIC approach [39]. This index $[\mathrm{PCMI}=114 \times \mathrm{I}($ Male $)+2 \times(100 \%-\mathrm{FVC} \%$ predicted $)+3 \times(100 \%-$ Dlco $\%$ predicted $)+111 \times \mathrm{I}($ MMP-7 $\geq 4.3 \mathrm{ng} / \mathrm{ml})]$ was highly predictive of mortality in the replication cohort with a $\mathrm{C}$-index for early mortality of 84 . Although it is hard to compare to other studies, this prediction seems to be substantially better than a recently proposed clinical staging system that achieved acceptable C-indexes of 69-72.3 for mortality prediction [40"], suggesting that the use of molecular markers will as expected improve upon clinical predictions in IPF.

Similarly, changes in circulating blood cell populations have been associated with outcome. Moeller et al. [41] demonstrated in a cohort of 51 patients that increases in circulating fibrocytes predicted poor prognosis and Gilani et al [42] observed that downregulation of CD28 in circulating CD4 T cells was a marker of poor prognoses in a cohort of 89 IPF patients. 
Overall, the outcome prediction based on the peripheral blood seems to hold significant promise. Our study, the largest so far and the first to have two cohorts, demonstrated the reproducibility of the markers as well as the feasibility of integrating clinical and molecular markers. Considering the significant evidence that patients with IPF may present on distinct outcome trajectories, it seems very important that all clinical studies will collect biomarker information as results may be affected by population stratification.

\section{DISEASE ACTIVITY MARKERS}

Disease activity markers are obviously not as developed as the two previous classes of biomarkers in IPF. At this stage, there is no real definition of the disease activity of IPF one could conceive that KL-6, SP-A and MMP-7 are markers of alveolar epithelial cell injury and CCL-18 a marker of alveolar macrophage activation, but strangely enough as yet we do not have markers for some of the processes that happen in IPF such as deposition of new collagen. Mechanistically, the biomarker that may be tied most closely to disease pathogenesis is MMP-7, a pluripotent matrix metalloprotease expressed in alveolar type II cells. MMP-7 is a WNT/ $\beta$-catenin pathway target molecule [43], suggesting that increases of MMP-7 are reflective of aberrant WNT/ $\beta$ catenin that has been described in IPF [44,45], MMP-7 knockout mice are relatively protected from bleomycin-induced fibrosis, suggesting that It is mechanistically involved in the fibrosis pathways [46]. However, so far, there is no data to support MMP-7 as a marker of disease activity.

Another way to look at the markers of disease activity is to identify the markers of acute exacerbations of IPF (AE-IPF), episodes of decline in respiratory status without an identifiable cause [47], that lead to significant mortality [48]. Of the previous markers mentioned, KL-6 has been mostly widely studied in this context $[8,49,50]$, It seems that AEIPF are associated with increases in blood KL-6, although the mechanisms are not clear. We compared the gene expression in the lungs of patients with AE-IPF lungs to stable IPF [51] and identified 579 differentially expressed genes, and did not find any indication of infectious or inflammatory cause. We did find an increase in a-defensins, a group of innate antimicrobial peptides, in the mRNA levels as well as in the plasma protein level of AE-IPF patients, suggesting that they should be evaluated as biomarkers for acute exacerbations [52].

\section{DRUG EFFICACY BIOMARKERS}

At this stage, there are no drug efficacy biomarkers in IPF. However, our knowledge of disease mechanisms as well the drugs that are now being evaluated may allow some speculation. For instance, one could advocate that markers of TGF- $\beta$ activation would be assessed in a study that aims to reduce the local TGF- $\beta$ [53], downstream effects of kinase inhibition would be assessed in a study that involves receptor tyrosine kinase inhibition [54] or that MMP-7 levels would be followed if a WNT inhibitor was studied (the authors do not know of such study). At this stage - all of these suggestions are speculative, but insistence by the scientific community on drug efficacy markers will greatly enhance our understanding of IPF, the mechanisms of action of certain drugs and, most importantly, 
whether drug studies fail because the drug actually is not effective or because of other confounders.

\section{CONCLUSION}

In this review, we presented the overwhelming evidence that the peripheral blood is highly informative with regard to disease presence, stage and outcome. The most significant advance in the last year is the addition of two sets of validated markers: one is the replicated gene association of MUC5B polymorphism with IPF, and the other is the derivation of the combined clinical and molecular outcome index (PCMI). In both cases, the strength lies in the relative size of the cohorts and the replication. However, it is critical to note that the majority of markers have not been formally replicated and that implementation of markers in drug studies and clinical practice will require a coordinated validation effort.

Standardization of methods, recruitment of large numbers of highly phenotyped individuals and relevant controls, including other chronic lung diseases, prospective longitudinal followup and sampling, and an integrated approach that incorporates validation with discovery will be required. This is a significant effort and obviously beyond the capacity of a single center; moreover, in the current funding atmosphere such an effort will not be sustainable if dependent on NIH funding. Thus, it is recommended that an IPF biomarker consortium consisting of industry, patient advocacy organizations and the scientific community should be organized. Under such a structure, companies and institutions will be able to contribute samples and clinical information for the validation of currently known biomarkers, while still maintaining the analysis of their favorite markers and benefitting from a much larger pool of patients. On the basis of a significant and impressive body of knowledge that was derived under less optimal research conditions, it seems that a community-wide study will indeed identify all classes of biomarkers and transform our research and management of IPF.

\section{Acknowledgments}

The work was supported by the NIH Grants U01HL108642, U01HL112707, RC2HL101715, R01HL095397 and R01LM009657.

\section{REFERENCES AND RECOMMENDED READING}

Papers of particular interest, published within the annual period of review, have been highlighted as:

- of special interest

- of outstanding interest

Additional references related to this topic can also be found in the Current World Literature section in this issue (pp. 537-538).

1. American Thoracic Society/European Respiratory Society. American Thoracic Society/European Respiratory Society International Multidisciplinary Consensus Classification of the idiopathic interstitial pneumonias. Am J Respir Crit Care Med. 2002; 165:277-304. [PubMed: 11790668] 
2. Fernandez Perez ER, Daniels CE, Schroeder DR, et al. Incidence, prevalence, and clinical course of idiopathic pulmonary fibrosis: a population-based study. Chest. 2010; 137:129-137. [PubMed: 19749005]

3. Raghu G, Collard HR, Anstrom KJ, et al. Idiopathic pulmonary fibrosis: clinically meaningful primary endpoints in phase 3 clinical trials. Am J Respir Crit Care Med. 2012; 185:1044-1048. [PubMed: 22505745]

4r. Raghu G, Collard HR, Egan JJ, et al. An official ATS/ERS/JRS/ALAT statement: idiopathic pulmonary fibrosis: evidence-based guidelines for diagnosis and management. Am J Respir Crit Care Med. 2011; 183:788-824. The most recent official evidence based guidelines for the diagnosis and management of IPF. [PubMed: 21471066]

5-. King TE Jr, Pardo A, Selman M. Idiopathic pulmonary fibrosis. Lancet. 2011; 378:1949-1961. An outstanding state-of-art review on IPF. [PubMed: 21719092]

6. Vij R, Noth I. Peripheral blood biomarkers in idiopathic pulmonary fibrosis. Transl Res. 2012; 159:218-227. [PubMed: 22424426]

7. Mazur W, Toljamo T, Ohlmeier S, et al. Elevation of surfactant protein A in plasma and sputum in cigarette smokers. Eur Respir J. 2011; 38:277-284. [PubMed: 21273386]

8. Ishikawa N, Hattori N, Yokoyama A, Kohno N. Utility of KL-6/MUC1 in the clinical management of interstitial lung diseases. Respir Investig. 2012; 50:3-13.

9. Prasse A, Stahl M, Schulz G, et al. Essential role of osteopontin in smoking-related interstitial lung diseases. Am J Pathol. 2009; 174:1683-1691. [PubMed: 19359522]

10. Rosas IO, Richards TJ, Konishi K, et al. MMP1 and MMP7 as potential peripheral blood biomarkers in idiopathic pulmonary fibrosis. PLoS Med. 2008; 5:e93. [PubMed: 18447576]

11. Vuorinen K, Myllamiemi M, Lammi L, et al. Elevated matrilysin levels in bronchoalveolar lavage fluid do not distinguish idiopathic pulmonary fibrosis from other interstitial lung diseases. APMIS. 2007; 115:969-975. [PubMed: 17696954]

12. Lung Genomics Resource Consortium. 2012. https://http://www.lung-genomics.org/auth/login? service $=\mathrm{https} \% 3 \mathrm{a} \% 2 \mathrm{f} \% 2 \mathrm{fwww}$. lung-genomics.org $\% 2 \mathrm{fresearch} \% 2 \mathrm{f}$

13. Thomas AQ, Lane K, Phillips J 3rd, et al. Heterozygosity for a surfactant protein C gene mutation associated with usual interstitial pneumonitis and cellular nonspecific interstitial pneumonitis in one kindred. Am J Respir Crit Care Med. 2002; 165:1322-1328. [PubMed: 11991887]

14. Lawson WE, Grant SW, Ambrosini V, et al. Genetic mutations in surfactant protein C are a rare cause of sporadic cases of IPF. Thorax. 2004; 59:977-980. [PubMed: 15516475]

15. Wang Y, Kuan PJ, Xing C, et al. Genetic defects in surfactant protein A2 are associated with pulmonary fibrosis and lung cancer. Am J Hum Genet. 2009; 84:52-59. [PubMed: 19100526]

16. Armanios MY, Chen JJ, Cogan JD, et al. Telomerase mutations in families with idiopathic pulmonary fibrosis. N Engl J Med. 2007; 356:1317-1326. [PubMed: 17392301]

17. Tsakiri KD, Cronkhite JT, Kuan PJ, et al. Adult-onset pulmonary fibrosis caused by mutations in telomerase. Proc Natl Acad Sci USA. 2007; 104:7552-7557. [PubMed: 17460043]

18. Mushiroda T, Wattanapokayakit S, Takahashi A, et al. Pirfenidone Clinical Study G. A genomewide association study identifies an association of a common variant in TERT with susceptibility to idiopathic pulmonary fibrosis. J Med Genet. 2008; 45:654-656. [PubMed: 18835860]

19. Cronkhite JT, Xing C, Raghu G, et al. Telomere shortening in familial and sporadic pulmonary fibrosis. Am J Respir Crit Care Med. 2003; 178:729-737. [PubMed: 18635888]

20. Alder JK, Chen JJ, Lancaster L, et al. Short telomeres are a risk factor for idiopathic pulmonary fibrosis. Proc Natl Acad Sci USA. 2008; 105:13051-13056. [PubMed: 18753630]

21. Hodgson U, Pulkkinen V, Dixon M, et al. ELMOD2 is a candidate gene for familial idiopathic pulmonary fibrosis. Am J Hum Genet. 2006; 79:149-154. [PubMed: 16773575]

22. Hutyrova B, Pantelidis P, Drabek J, et al. Interleukin-1 gene cluster polymorphisms in sarcoidosis and idiopathic pulmonary fibrosis. Am J Respir Crit Care Med. 2002; 165:148-151. [PubMed: 11790645]

23. Zorzetto M, Ferrarotti I, Trisolini R, et al. Complement receptor 1 gene polymorphisms are associated with idiopathic pulmonary fibrosis. Am J Respir Crit Care Med. 2003; 168:330-334. [PubMed: 12773320] 
24. Latsi P, Pantelidis P, Vassilakis D, et al. Analysis of IL-12 p40 subunit gene and IFN-gamma G5644A polymorphisms in idiopathic pulmonary fibrosis. Respir Res. 2003; 4:6. [PubMed: 12914676]

25. Zorzetto M, Ferrarotti I, Campo I, et al. NOD2/CARD15 gene polymorphisms in idiopathic pulmonary fibrosis. Sarcoidosis Vasc Diffuse Lung Dis. 2005; 22:180-185. [PubMed: 16315780]

26. Checa M, Ruiz V, Montano M, et al. MMP-1 polymorphisms and the risk of idiopathic pulmonary fibrosis. Hum Genet. 2008; 124:465-472. [PubMed: 18853190]

27. Liu L, Dai HP, Xiao B, et al. Association of ENA 78, IPp-10 and VEGF gene polymorphism with idiopathic pulmonary fibrosis. Zhonghua Yi Xue Za Zhi. 2009; 89:2690-2694. [PubMed: 20137269]

28. Boumazos S, Boumazou I, Murchison JT, et al. Fcgamma receptor IIIb (CD16b) polymorphisms are associated with susceptibility to idiopathic pulmonary fibrosis. Lung. 2010; 188:475-481. [PubMed: 20924590]

29. Ahn MH, Park BL, Lee SH, et al. A promoter SNP rs4073T >A in the common allele of the interleukin 8 gene is associated with the development of idiopathic pulmonary fibrosis via the IL-8 protein enhancing mode. Respir Res. 2011; 12:73. [PubMed: 21649933]

30. Martinclli M, Pacilli AM, Rivetti S, et al. A role for epidermal growth factor receptor in idiopathic pulmonary fibrosis onset. Mol Biol Rep. 2011; 38:4613-4617. [PubMed: 21132379]

31.. Seibold MA, Wise AL, Speer MC, et al. A common MUC5b promoter polymorphism and pulmonary fibrosis. N Engl J Med. 2011; 364:1503-1512. The first report of a significant association of MUC5B polymorphism with IPF. [PubMed: 21506741]

32. Zhang Y, Noth I, Garcia JG, Kaminski N. A variant in the promoter of MUC5b and idiopathic pulmonary fibrosis. N Engl J Med. 2011; 364:1576-1577. [PubMed: 21506748]

33. Selman M, Carrillo G, Estrada A, et al. Accelerated variant of idiopathic pulmonary fibrosis: clinical behavior and gene expression pattern. PLoS One. 2007; 2:e482. [PubMed: 17534432]

34. Boon K, Bailey NW, Yang J, et al. Molecular phenotypes distinguish patients with relatively stable from progressive idiopathic pulmonary fibrosis (IPF). PLoS One. 2009; 4:e5134. [PubMed: 19347046]

35. Prasse A, Probst C, Bargagli E, et al. Serum CC-chemokine ligand 18 concentration predicts outcome in idiopathic pulmonary fibrosis. Am J Respir Crit Care Med. 2009; 179:717-723. [PubMed: 19179488]

36. Kinder BW, Brown KK, McCormack FX, et al. Serum surfactant protein-A is a strong predictor of early mortality in idiopathic pulmonary fibrosis. Chest. 2009; 135:1557-1563. [PubMed: 19255294]

37. Korthagen NM, van Moorsel CH, Barlo NP, et al. Serum and BALF YKL-40 levels are predictors of survival in idiopathicpulmonary fibrosis. Respir Med. 2011; 105:106-113. [PubMed: 20888745]

38-.. Richards TJ, Kaminski N, Baribaud F, et al. Peripheral blood proteins predict mortality in idiopathic pulmonary fibrosis. Am J Respir Crit Care Med. 2012; 185:67-76. So far, the largest and most extensive study of makers that predict outcome in IPF. Combination of clinical and molecular markers of significant interest. [PubMed: 22016448]

39. Venables, WN.; Ripley, BD. Modern applied statistics with S. 4. New York: Springer; 2002.

40-. Ley B, Ryerson CJ, Vittinghoff E, et al. A multidimensional index and staging system for idiopathic pulmonary fibrosis. Ann Intern Med. 2012; 156:684-691. An ambitious and elegant study aiming to develop a staging system for IPF. [PubMed: 22586007]

41. Moeller A, Gilpin SE, Ask K, et al. Circulating fibrocytes are an indicator of poor prognosis in idiopathic pulmonary fibrosis. Am J Respir Crit Care Med. 2009; 179:588-594. [PubMed: 19151190]

42. Gilani SR, Vuga U, Lindell KO, et al. CD28 down regulation on circulating CD4 T cells is associated with poor prognoses of patients with idiopathic pulmonary fibrosis. PLoS One. 2010; 5:e8959. [PubMed: 20126467]

43. He W, Tan RJ, Li Y, et al. Matrix metalloproteinase-7 as a surrogate marker predicts renal WNT/ beta-catenin activity in CKD. J Am Soc Nephrol. 2012; 23:294-304. [PubMed: 22095947] 
44. Chilosi M, Poletti V, Zamo A, et al. Aberrant WNT/beta-catenin pathway activation in idiopathic pulmonary fibrosis. Am J Pathol. 2003; 162:1495-1502. [PubMed: 12707032]

45. Konigshoff M, Kramer M, Balsara N, et al. WNT1 -inducible signaling protein-1 mediates pulmonary fibrosis in mice and is upregulated in humans with idiopathic pulmonary fibrosis. $\mathbf{J}$ Clin Invest. 2009; 119:772-787. [PubMed: 19287097]

46. Zuo F, Kaminski N, Eugui E, et al. Gene expression analysis reveals matrilysin as a key regulator of pulmonary fibrosis in mice and humans. Proc Natl Acad Sci USA. 2002; 99:6292-6297. [PubMed: 11983918]

47. Collard HR, Moore BB, Flaherty KR, et al. Acute exacerbations of idiopathic pulmonary fibrosis. Am J Respir Crit Care Med. 2007; 176:636-643. [PubMed: 17585107]

48. Song JW, Hong SB, Lim CM, et al. Acute exacerbation of idiopathic pulmonary fibrosis: incidence, risk factors and outcome. Eur Respir J. 2011; 37:356-363. [PubMed: 20595144]

49. Collard HR, Calfee CS, Wolters PJ, et al. Plasma biomarker profiles in acute exacerbation of idiopathic pulmonary fibrosis. Am J Physiol Lung Cell Mol Physiol. 2010; 299:L3-L7. [PubMed: 20418386]

50. Satoh H, Kurishima K, Ishikawa H, Ohtsuka M. Increased levels of KL-6 and subsequent mortality in patients with interstitial lung diseases. J Intern Med. 2006; 260:429-434. [PubMed: 17040248]

51. Konishi K, Gibson KF, Lindell KO, et al. Gene expression profiles of acute exacerbations of idiopathic pulmonary fibrosis. Am J Respir Crit Care Med. 2009; 180:167-175. [PubMed: 19363140]

52. Zasloff M. Antimicrobial peptides of multicellular organisms. Nature. 2002; 415:389-395. [PubMed: 11807545]

53. STX-100 in patients with idiopathic pulmonary fibrosis (IPF). 2012. http://clinicaltrials.gov/ct2/ show/NCT01371305?term $=I P F+$ stromedix \&rank $=1$. stromedix \&rank $=1$

54. Safety and efficacy of BIBF 1120 at high dose in idiopathic pulmonary fibrosis patients. 2012. http://clinicaltrials.gov/ct2/show/NCT01335464?term=IPF+BIBF\&rank=3 


\section{KEY POINTS}

- IPF is a chronic progressive and frequently lethal interstitial lung disease with an unpredictable course.

- The multiple studies that identified potential diagnostic and prognostic markers in the peripheral blood were limited in size and lacked replication but together suggested a strong outcome signal in the blood.

- A recently discovered MUC5B SNP is strongly associated with IPF and has been replicated in several cohorts.

- Recently, a personal clinical and molecular outcome index (PCMl) that integrates clinical parameters and MMP-7 plasma concentrations and accurately predicts outcome was derived and validated.

- The success of recent studies suggests that outcome prediction in IPF can be improved using peripheral blood biomarkers and thus they should be incorporated in drug study design and evaluated for the use for transplant prioritization. 